\title{
Rapidly progressive metastatic cholangiocarcinoma in a postpartum patient with cystic fibrosis: a case report
}

\author{
Sara W. Carson ${ }^{1 *}$ D, Kelly E. Craven², David Nauen², Kristina Montemayor ${ }^{1}$, Mark Yarchoan ${ }^{3}$, William R. Burns ${ }^{4}$,
} Christian A. Merlo ${ }^{1,5}$ and Natalie E. West ${ }^{1}$

\begin{abstract}
Background: Cholangiocarcinoma is a rare gastrointestinal malignancy that arises within the intrahepatic, perihilar, and/or extrahepatic bile ducts. Individuals with cystic fibrosis are at increased risk for gastrointestinal malignancies. The most common gastrointestinal malignancy in cystic fibrosis is colon cancer, but other gastrointestinal malignancies also occur at greater rates than the general population.

Case presentation: We present a case of a rapidly progressive metastatic intrahepatic cholangiocarcinoma in an individual with cystic fibrosis who was 5 months postpartum, incidentally found while undergoing a lung transplantation evaluation.

Conclusion: A heightened clinical awareness of gastrointestinal malignancies, beyond colon cancer, in individuals with cystic fibrosis is warranted. It remains unclear if pregnancy is an additional risk factor for gastrointestinal malignancies in cystic fibrosis.
\end{abstract}

Keywords: Cholangiocarcinoma, Gastrointestinal cancer, Cystic fibrosis, pregnancy, Case report

\section{Background}

Individuals with cystic fibrosis (CF) are at higher risk for digestive tract cancers $[1,2]$. Cystic fibrosis transmembrane conductance regulator (CFTR)-regulated chloride channels are highly expressed in the gastrointestinal tract and when disrupted, as in CF, there is increased inflammation and cell turnover, which is believed to contribute to this increased risk [3, 4]. Furthermore, there is limited evidence that pregnancy may decrease the maternal immune system and thus increase the risk of, or at least increase the progression of malignancy [5-8]. However, it is unknown if pregnancy itself would have an additive effect on the increased risk of digestive tract

\footnotetext{
* Correspondence: scarson6@jhmi.edu

'Department of Medicine, Division of Pulmonary and Critical Care Medicine, Johns Hopkins University, 1830 E. Monument St 5th Floor, Baltimore, MD 21205, USA

Full list of author information is available at the end of the article
}

cancers in women with CF. We report a case of a woman with CF who was 5 months postpartum who was diagnosed with rapidly progressive metastatic intrahepatic cholangiocarcinoma incidentally discovered during evaluation for lung transplantation.

\section{Case presentation}

A 26-year-old female with CF (genotype F508del/ F508del), with a baseline forced expiratory volume in $1 \mathrm{~s}$ $\left(\mathrm{FEV}_{1}\right)$ of $21 \%$ predicted and a $6-1$ home oxygen requirement, was undergoing evaluation for bilateral lung transplantation. Her medical history included recurrent respiratory infections with multi-drug resistant pathogens, pancreatic insufficiency, malnutrition requiring percutaneous gastrostomy feeds, atrioventricular nodal reentry tachycardia status post ablation, and the recent delivery of a healthy baby. She had no history of biliary tract stones, 
cirrhosis, hepatitis B, or hepatitis C. As part of the lung transplantation workup a CT scan of the chest, abdomen, and pelvis revealed a new peripherally enhancing $6.3 \mathrm{~cm}$ mass in the right lobe of her liver, and subsequent MRI suggested intrahepatic cholangiocarcinoma. At this time, the patient reported feeling a hard mass under her right ribs along with intermittent right upper quadrant pain and fullness, anorexia, weight loss, and daily subjective fevers for several weeks.

Ultrasound-guided biopsy identified the mass as an adenocarcinoma, with positive staining for cytokeratin 7 (CK7), GATA-3, a transcription factor important in the regulation of certain genes, and p40, a marker of squamous differentiation. Notably, the tumor was negative for estrogen receptor (ER), thyroid transcription factor 1 (TTF-1), cytokeratin 20 (CK20), and high risk human papillomavirus ribonucleic acid in situ hybridization (HR HPV RNA ISH), and therefore did not support tumor origin of breast or gynecologic, lung, lower gastrointestinal, or cervical or head/neck, respectively. Serum tumor markers demonstrated normal alpha-fetoprotein (AFP) and carcinoembryonic antigen (CEA) levels, but a markedly elevated carbohydrate antigen (CA)19-9 level of $5949.5 \mathrm{U} / \mathrm{mL}(0-36 \mathrm{U} / \mathrm{mL})$, which can be elevated in pancreatic, gastric, hepatobiliary, and colonic malignancies. Given the tumor location, serum markers, and pathology findings, the tumor was thought to be most consistent with a primary cholangiocarcinoma.

The patient underwent complete staging following hospital discharge, where radiation therapy with curative intent was recommended. Ultimately her poor performance status, chronic pulmonary disease, and risk of severe life-threatening infection influenced the decision not to pursue systemic chemotherapy or surgery as the initial treatment modality. However, prior to starting treatment, she developed pneumonia leading to acute respiratory failure requiring intubation. A second CT of the chest, abdomen, and pelvis (4 weeks from the initial CT) revealed the liver mass had grown to $13.6 \times 10 \mathrm{~cm}$ and there were patchy pulmonary consolidations concerning for both pneumonia and metastatic disease. Positron emission tomography (PET) scan demonstrated uptake in the lungs bilaterally, mediastinal lymph nodes, right iliac, and right femur, consistent with metastatic disease. Given her critical condition, it was decided she was no longer a candidate for treatment of her cancer. She was transitioned to home hospice where she passed away approximately 10 weeks after the initial CT.

Autopsy revealed a $20 \times 17 \times 23 \mathrm{~cm}$ tan mass in the right lobe of the liver (Fig. 1a and b), consistent with intrahepatic cholangiocarcinoma (Fig. 1c), along with metastatic deposits in the heart/epicardium (Fig. 1d), bilateral lungs (Fig. 1e), anterior/posterior cul-de-sacs, left ovary, vaginal adventitia, spleen, diaphragm and bone marrow (Fig. 1f).

Intrahepatic cholangiocarcinoma can resemble adenocarcinomas from other sites, and because there are no histologic markers that can reliably make the distinction, it is often difficult to distinguish them from metastatic tumors [9]. Due to a lack of a dominant tumor at other possible primary sites at autopsy, and a morphology showing anastomosing glands in a fibrotic stroma with focal squamous differentiation, cholangiocarcinoma was favored in this case.

\section{Discussion}

The median life expectancy of individuals with CF has increased significantly over the past few decades, now estimated to be 47.4 years of those born in 2018 [10]. This longer life expectancy has resulted in an increased incidence of some types of cancer among those with CF $[1,2,11]$.

Individuals with CF have an increased risk of gastrointestinal cancers, the most common being colon cancer $[1,2]$. The first reported case of cholangiocarcinoma in a patient with CF was described by Abdul-Karim in 1982. The patient had a history of CF, recurrent pulmonary infections, pancreatic insufficiency, and biliary tract stones [12]. Since then, to our knowledge, there have been fewer than 10 case reports of biliary tract cancer in those with CF who have not undergone organ transplant [1214]. In 2003, Maisonneuve and colleagues conducted a 10 -year nationwide epidemiologic study that looked at the risk of cancer in non-transplanted and transplanted $\mathrm{CF}$ patients and found that both non-transplanted and transplanted CF patients were at greater risk of gastrointestinal cancers, including biliary tract cancers, compared to the age-adjusted population [15]. In 2013, the same authors conducted a 20-year nationwide epidemiologic study to evaluate the risk of cancer in CF and found there was a 3.5-fold increase in all digestive tract cancers compared to the age-adjusted general population. Specifically, the standardized incidence ratios (SIR) for colon cancer was $6.2(95 \% \mathrm{CI}=4.2$ to 9.0$)$, for small bowel cancer was 11.5 (95\% CI 4.2 to 25.4), and for biliary tract cancer was 11.4 (95\% CI 3.6 to 27.4) [2]. A recent systematic review and meta-analysis from 2018 reported even greater SIRs for these cancers and found that those with CF who received an organ transplant had a 2-5 times increased risk of developing gastrointestinal cancers compared to those who did not [1].

CFTR-regulated chloride channels, highly expressed in many of the gastrointestinal organs, are known to play an instrumental role in the digestive tract. Therefore, individuals with $\mathrm{CF}$ are at greater risk of developing a range of gastrointestinal tract dysfunctions. These include deficient anion (Cl- and $\mathrm{HCO}_{-}$) and fluid transport, impaired release and clearance of mucus which can 


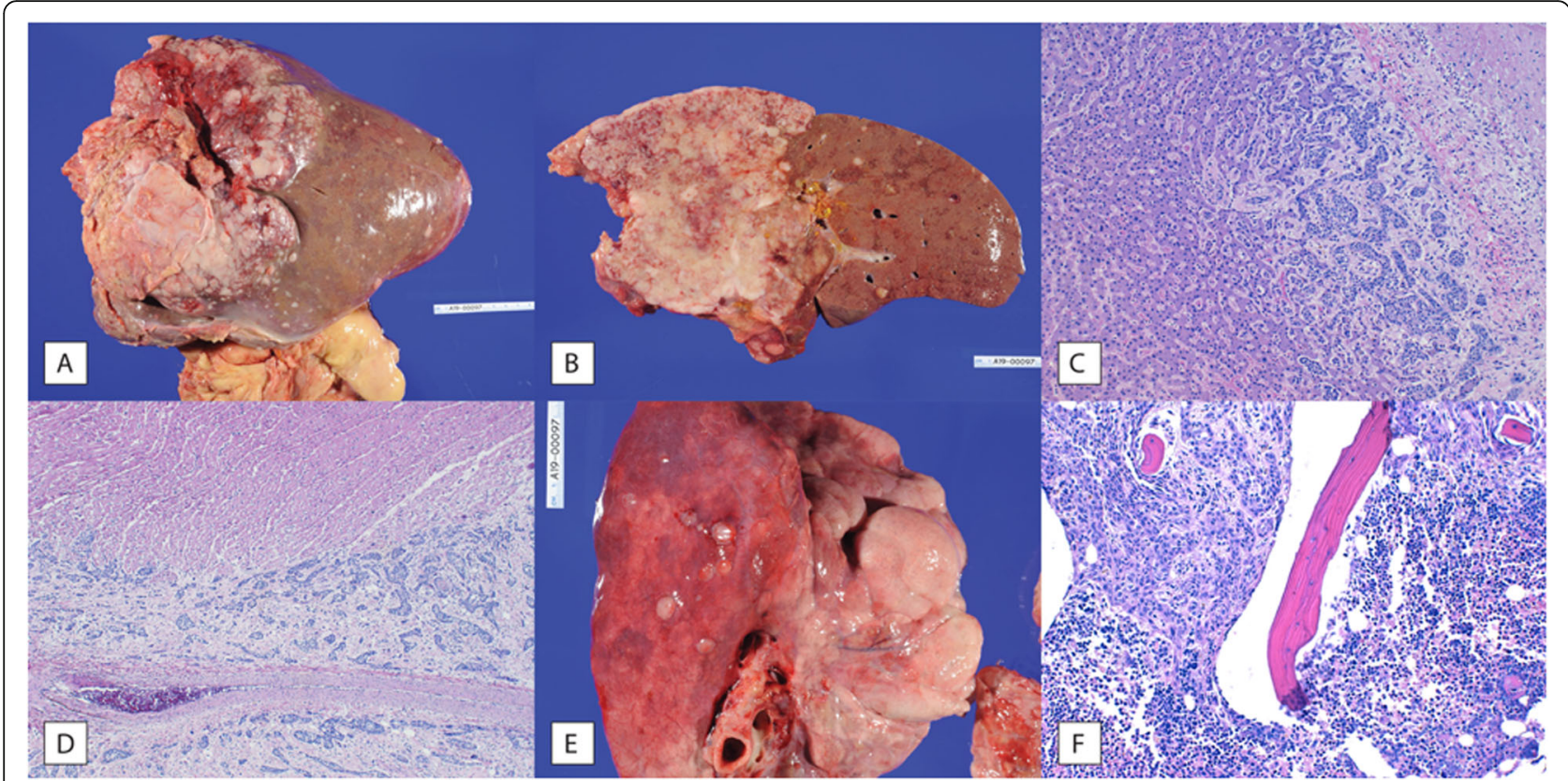

Fig. 1 Metastatic Cholangiocarcinoma. a Liver with grossly visible tan mass measuring $23 \times 20 \times 17 \mathrm{~cm}$ primarily involving the right lobe and multiple satellite lesions. $\mathbf{b}$ Cross-section of the liver demonstrating the mass and multiple satellite lesions. $\mathbf{c}$ A satellite lesion within the liver shows nests of cancerous cells with round to oval nuclei within a fibrotic stroma (right) invading into adjacent normal hepatocyte trabeculae and sinusoids (left). d Epicardial and myocardial invasion of metastatic cholangiocarcinoma. Representative section shows nests of tumor cells within a fibrotic stroma around a large vessel (bottom) invading into cardiac myocytes (top). e Lung with multiple tan-pink metastatic deposits on the visceral pleura. $\mathbf{f}$ Bone marrow involvement by metastatic cholangiocarcinoma. Normal trilineage hematopoiesis involving erythroid precursors, myeloid precursors, and megakaryocytes to the right of the bone spicule is replaced by solid/trabecular areas of tumor cells to the left of the bone spicule

lead to meconium ileus and distal intestinal obstructive syndrome (DIOS), pancreatic insufficiency, decreased intestinal lumen $\mathrm{pH}$, increased intestinal stem cell $\mathrm{pH}$, abnormal bacterial colonization, microbial dysbiosis, and impaired innate immune responses that lead to chronic inflammation $[3,16]$. As a result, CF individuals have increased chronic intestinal inflammation and intestinal cell turnover which are believed to increase the risk of gastrointestinal cancers among CF individuals [3, 4]. In addition, CFTR is believed to be a tumor suppressor gene and disruption, as in $\mathrm{CF}$, leads to increased risk for colon cancer and other digestive tract cancers $[17,18]$.

Cholangiocarcinoma refers to cancers of the bile duct that arise in the intrahepatic $(<10 \%$ of cases), perihilar, or distal (extrahepatic) biliary tree [19]. Cholangiocarcinoma is rare, with a reported incidence of 2 cases per 100,000 in the US and represents approximately $3 \%$ of all gastrointestinal malignancies [20]. Risk factors include chronic biliary tract inflammation due to primary sclerosing cholangitis, choledochal cysts, chronic cholelithiasis/choledocholithiasis, hepatolithiasis, chronic viral and non-viral liver disease, infections such as HIV, Helicobacter pylori, and certain parasitic infections, obesity, medications, $\mathrm{CF}$, and several other genetic conditions such as Lynch syndrome, multiple biliary papillomatosis, and
BAP1 tumor predisposition syndrome $[1,13,21,22]$. These risk factors all lead to chronic inflammation and/or cholestasis in the bile duct, which change the microenvironment in the bile duct, increasing the risk of cholangiocarcinoma [23]. In addition, it is likely that there are also successive genetic abnormalities contributing to the pathogenesis involving both oncogenes (RAS, ERBB2, BRAF, EGFR, PIK3CA, CTNNB1) and tumor suppressor genes (e.g. p53, SMAD4, CDKN2A) [24-26].

Other than CF, our patient did not have any known risk factors for cholangiocarcinoma that have been previously described in the literature. However, she was 5 months postpartum at the time of her cancer diagnosis. Although we cannot be certain as to whether the cholangiocarcinoma was present before her pregnancy, this is of particular interest as Qasrawi and colleagues reported a case of cholangiocarcinoma discovered during pregnancy and compared their case to 9 other similar cases in the literature where cholangiocarcinoma was diagnosed during pregnancy [5]. AFP and placental steroids may be responsible for immune system suppression during pregnancy $[6,7]$. Furthermore, more aggressive neoplasia in pregnant women is thought to be correlated to the suppression of maternal immunity. The fetus may act as a natural allograft and the 
nonrejection mechanism that operates in malignancy may also be triggered in pregnancy, making a tumor or a fetus acceptable to the host and may contribute to development or progression of malignancy in susceptible individuals [8]. Data for this proposed mechanism are limited; however, and this theory remains mainly speculative. Although the association between our patient with CF who was pregnant and subsequently was diagnosed with cholangiocarcinoma is of scientific interest, there are only a limited number of reported cases of cholangiocarcinoma cases in pregnancy in the literature and none in CF that we know of. It remains unclear if pregnancy has an additive effect on the risk of development and progression of cholangiocarcinoma among those with CF.

In conclusion, our case demonstrates a unique presentation of a rapidly progressing intrahepatic cholangiocarcinoma in an individual with cystic fibrosis who was 5 months postpartum undergoing lung transplantation workup. It remains unclear as to whether the presentation of her advanced disease was due to immunosuppression of pregnancy or delayed diagnosis because of the pregnancy itself. Our case, supported by the literature, suggests that a heightened clinical awareness for the increased risk of gastrointestinal cancers, beyond colon cancer, and in pregnancy, is warranted in CF.

\section{Abbreviations}

CF: Cystic fibrosis; FEV 1 : Forced expiratory volume in $1 \mathrm{~s}$; CK7: Cytokeratin 7; ER: Estrogen receptor; TTF-1: Thyroid transcription factor 1; CK20: Cytokeratin 20; HR HPV RNA ISH: High risk human papillomavirus ribonucleic acid in situ hybridization; AFP: Alpha-fetoprotein; CEA: Carcinoembryonic antigen; CA-199: Carbohydrate antigen 19-9; PET: Positron emission tomography; SIR: Standardized incidence ratios; CFTR: Cystic fibrosis transmembrane conductance regulator; DIOS: Distal intestinal obstructive syndrome

\section{Acknowledgements}

The authors would like to thank Jeanne S Sheffield, M.D., a professor of Gynecology and Obstetrics at Johns Hopkins University, and expert in Maternal-Fetal Medicine, for her contributions to this manuscript. The authors would also like to thank our patient's family for allowing us to share her case in the literature.

\section{Authors' contributions}

SWC and NEW contributed to the conception and authorship of this manuscript. NEW personally cared for this patient for many years and provided details regarding her clinical course. KEC and DN provided their expert opinion regarding the pathology results, drafted portions of this manuscript as well as provided substantial revisions. KM provided substantial revisions to the manuscript. MY personally cared for this patient and provided expertise regarding the unique presentation of her malignancy and also provided substantial revisions to this manuscript. WRB personally cared for this patient and provided his expert opinion in treatment for this patient as well as provided substantial revisions to the manuscript. CAM personally cared for this patient, provided details regarding her clinical course, and provided substantial revisions to the manuscript. All authors reviewed and approved the manuscript prior to submission for this publication.

\section{Funding}

This work was supported by the National Heart, Lung, And Blood Institute of the NIH award number T32HL007534(SWC) and WEST17Y5 (NEW). Both sources of funding provided salary support that allowed for the creation of this manuscript.
Availability of data and materials

Not applicable.

Ethics approval and consent to participate

Not applicable.

\section{Consent for publication}

Written consent was obtained from the patient's parents for the publication of this manuscript.

\section{Competing interests}

The authors have no conflicts of interest to report that is related to this work.

\section{Author details}

'Department of Medicine, Division of Pulmonary and Critical Care Medicine, Johns Hopkins University, 1830 E. Monument St 5th Floor, Baltimore, MD 21205, USA. Department of Pathology, Johns Hopkins University, Baltimore, MD, USA. ${ }^{3}$ Department of Oncology, Johns Hopkins University, Baltimore, MD, USA. ${ }^{4}$ Department of Surgery, Division of Surgical Oncology, Johns Hopkins University, Baltimore, MD, USA. ${ }^{5}$ Department of Epidemiology, Johns Hopkins Bloomberg School of Public Health, Baltimore, MD, USA.

Received: 17 September 2020 Accepted: 3 November 2020

Published online: 16 November 2020

\section{References}

1. Yamada A, Komaki Y, Komaki F, Micic D, Zullow S, Sakuraba A. Risk of gastrointestinal cancers in patients with cystic fibrosis: a systematic review and meta-analysis. Lancet Oncol. 2018;19(6):758-67.

2. Maisonneuve P, Marshall BC, Knapp EA, Lowenfels AB. Cancer risk in cystic fibrosis: a 20-year nationwide study from the United States. J Natl Cancer Inst. 2013;105(2):122-9.

3. Strubberg AM, Liu J, Walker NM, Stefanski CD, MacLeod RJ, Magness ST, et al. Cftr modulates Wnt/ $\beta$-catenin signaling and stem cell proliferation in murine intestine. Cell Mol Gastroenterol Hepatol. 2018;5(3):253-71.

4. Abraham JM, Taylor CJ. Cystic fibrosis \& disorders of the large intestine: DIOS, constipation, and colorectal cancer. J Cyst Fibros. 2017;16:540-9.

5. Qasrawi A, Abughanimeh O, Abu Ghanimeh M, Arora-Elder S, Yousef O, Tamimi T. Intrahepatic Cholangiocarcinoma Masquerading as Acute Fatty Liver of Pregnancy: A Case Report and Review of the Literature. Case Rep Hepatol. 2018; 26 [cited 2020 Jun 25];2018. Available from: https://www. ncbi.nlm.nih.gov/pmc/articles/PMC5846373/.

6. Murgita RA. The immunosuppressive role of alpha-fetoprotein during pregnancy. Scand J Immunol. 1976;5(9):1003-14.

7. Rembiesa R, Ptak W, Bubak M. The immunosuppressive effects of mouse placental steroids. Experientia. 1974;30(1):82-3.

8. Lau WY, Leung WT, Ho S, Lam SK, Li CY, Johnson PJ, et al. Hepatocellular carcinoma during pregnancy and its comparison with other pregnancyassociated malignancies. Cancer. 1995;75(11):2669-76.

9. Washington K. Masses of the liver. In: Sternberg's diagnostic surgical pathology. Sixth. Philadelphia, PA: Wolters Kluwer Health; 2015.

10. Cystic Fibrosis Foundation Patient Registry. 2018 Annual Data Report. Bethesda, Maryland. O2019 Cystic Fibrosis Foundation.

11. Sheldon CD, Hodson ME, Carpenter LM, Swerdlow AJ. A cohort study of cystic fibrosis and malignancy. Br J Cancer. 1993;68(5):1025-8.

12. Abdul-Karim FW, King TA, Dahms BB, Gauderer MW, Boat TF. Carcinoma of extrahepatic biliary system in an adult with cystic fibrosis. Gastroenterology. 1982:82(4):758-62

13. Perdue DG, Cass OW, Milla C, Dunitz J, Jessurun J, Sharp HL, et al. Hepatolithiasis and cholangiocarcinoma in cystic fibrosis: a case series and review of the literature. Dig Dis Sci. 2007;52(10):2638-42.

14. Tesluk H, McCauley K, Kurland G, Ruebner BH. Cholangiocarcinoma in an adult with cystic fibrosis. J Clin Gastroenterol. 1991;13(4):485-7.

15. Maisonneuve P, FitzSimmons SC, Neglia JP, Campbell PW, Lowenfels AB. Cancer risk in nontransplanted and transplanted cystic fibrosis patients: a 10-year study. J Natl Cancer Inst. 2003;95(5):381-7.

16. De Lisle RC, Borowitz D. The cystic fibrosis intestine. Cold Spring Harb Perspect Med. 2013;3(9):a009753.

17. Anderson KJ, Cormier RT, Scott PM. Role of ion channels in gastrointestinal cancer. World J Gastroenterol. 2019;25(38):5732-72. 
18. Than BLN, Linnekamp JF, Starr TK, Largaespada DA, Rod A, Zhang Y, et al. CFTR is a tumor suppressor gene in murine and human intestinal cancer. Oncogene. 2016;35(32):4191-9.

19. DeOliveira ML, Cunningham SC, Cameron JL, Kamangar F, Winter JM, Lillemoe KD, et al. Cholangiocarcinoma: thirty-one-year experience with 564 patients at a single institution. Ann Surg. 2007;245(5):755-62.

20. Siegel RL, Miller KD, Jemal A. Cancer statistics, 2019. CA Cancer J Clin. 2019; 69(1):7-34.

21. Chapman RW. Risk factors for biliary tract carcinogenesis. Ann Oncol Off J Eur Soc Med Oncol. 1999;10(Suppl 4):308-11.

22. Welzel TM, Mellemkjaer L, Gloria G, Sakoda LC, Hsing AW, El Ghormli L, et al. Risk factors for intrahepatic cholangiocarcinoma in a low-risk population: a nationwide case-control study. Int J Cancer. 2007;120(3):638-41.

23. Khan SA, Toledano MB, Taylor-Robinson SD. Epidemiology, risk factors, and pathogenesis of cholangiocarcinoma. HPB. 2008;10(2):77-82.

24. Nehls O, Gregor M, Klump B. Serum and bile markers for cholangiocarcinoma. Semin Liver Dis. 2004;24(2):139-54.

25. Isa T, Tomita S, Nakachi A, Miyazato H, Shimoji H, Kusano T, et al. Analysis of microsatellite instability, K-ras gene mutation and p53 protein overexpression in intrahepatic cholangiocarcinoma. Hepatogastroenterology. 2002;49(45):604-8.

26. Hezel AF, Deshpande V, Zhu AX. Genetics of biliary tract cancers and emerging targeted therapies. J Clin Oncol Off J Am Soc Clin Oncol. 2010; 28(21):3531-40.

\section{Publisher's Note}

Springer Nature remains neutral with regard to jurisdictional claims in published maps and institutional affiliations.

Ready to submit your research? Choose BMC and benefit from:

- fast, convenient online submission

- thorough peer review by experienced researchers in your field

- rapid publication on acceptance

- support for research data, including large and complex data types

- gold Open Access which fosters wider collaboration and increased citations

- maximum visibility for your research: over $100 \mathrm{M}$ website views per year

At $\mathrm{BMC}$, research is always in progress.

Learn more biomedcentral.com/submissions 